\title{
Efficacy and feasibility of the immunomagnetic separation based diagnosis for detecting sentinel lymph node metastasis from breast cancer
}

This article was published in the following Dove Press journal:

International Journal of Nanomedicine

7 April 2015

Number of times this article has been viewed

\author{
Xiang-Cheng Zhi ${ }^{1-3, *}$ \\ Min Zhang ${ }^{1-3, *}$ \\ Ting-Ting Meng ${ }^{1-3, *}$ \\ Xiao-Bei Zhang ${ }^{1-3}$ \\ Zhen-Dong Shi ${ }^{1-3}$ \\ Yan Liu ${ }^{1-3}$ \\ Jing-Jing Liu ${ }^{1-3}$ \\ Sheng Zhang ${ }^{1-3}$ \\ Jin Zhang ${ }^{1-3}$
}

'Third Department of Breast Cancer, People's Republic of China Tianjin Breast Cancer Prevention, Treatment and Research Center, Tianjin Medical University Cancer Institute and Hospital, National Clinical Research Center of Cancer, Tianjin, People's Republic of China; ${ }^{2}$ Key Laboratory of Breast Cancer Prevention and Therapy of the Ministry of Education, Tianjin, People's Republic of China; ${ }^{3}$ Key Laboratory of Cancer Prevention and Therapy, Tianjin, People's Republic of China

*These authors contributed equally to this work

Correspondence: Jin Zhang

Third Department of Breast Cancer People's Republic of China Tianjin Breast Cancer Prevention, Treatment and Research Center, Tianjin Medical University Cancer Institute and Hospital, West Beihuanhu Road, Tianjin 300060,

People's Republic of China

Tel +86 2223340123

Fax +86 2223340123

Email zhangin@timuch.com
Abstract: A purpose of this study was to establish a novel molecular diagnostic model and provide new insight into the intraoperative evaluation of the sentinel lymph node (SLN) metastasis in breast cancer. A total of 124 breast cancer patients who met the criteria of sentinel lymph node biopsy (SLNB) and underwent intraoperative biopsy were consecutively enrolled in this study. After the SLNs obtained from each patient were labeled, MOC-31 monoclonal antibody-mediated immunomagnetic separation (IMS) and flow cytometry were used to determine the expressions of breast cancer metastasis-related markers, including Mucin 1 (MUC1), CD44v6, and HER2. Alternatively, conventional intraoperative hematoxylin and eosin (HE) staining and cytokeratin immunohistochemistry (CK-IHC) were performed to detect potential SLN metastasis. The sensitivity, specificity, and false-negative rate of the three intraoperative diagnostic methods were compared and analyzed. A total of 55 positive-SLNs were found in 38 breast cancer patients using IMS, yielding a sensitivity of $86.4 \%$ (38/44), specificity of $94.7 \%$ (36/38), accuracy of $93.5 \%(116 / 124)$, false-positive rate of $2.5 \%(2 / 80)$, false-negative rate of $13.6 \%$ (6/44), positive predictive value of $95.5 \%$ (42/44), and negative predictive value of $93.0 \%(80 / 86)$. Patients with high expressions of CD44v6, MUC1, and HER2 in SLNs tended to have higher number of positive lymph nodes, among which the MUC1 and HER2 showed significant differences $(P<0.05)$. Therefore, compared with conventional HE staining and CK-IHC, IMS technology has remarkably higher sensitivity and specificity and relative lower false-negative rate, thus making it an effective and feasible intraoperative detection method of SLN for breast cancer diagnosis to some extent.

Keywords: immunomagnetic separation, breast cancer, sentinel lymph node, MUC1, CD44v6, HER2

\section{Introduction}

The surgical treatment strategy for breast cancer has shifted from "maximum tolerable" to "minimum effective." As a minimally invasive surgical technique for assessing the regional lymph node metastasis from malignancies, sentinel lymph node biopsy (SLNB) has been one of the standard procedures for breast cancer. ${ }^{1-3}$ For example, the NSABP B-32 enrolled 5,611 patients with clinically negative axillary lymph nodes and operable invasive breast cancer. Their updated follow-up data in 2011 showed that sentinel lymph node (SLN)-negative patients received simple follow-up or conventional axillary lymph node dissection (ALND), and their local control rate, disease-free survival, and overall survival showed no significant differences. ${ }^{4}$ Based on the findings of many randomized controlled trials, the most recent version of the NCCN Guidelines (2014) recommended that SLNB should be the choice for staging the axillary lymph nodes in patients with phase I or phase II breast cancer in settings where the medical staff have rich experiences in SLNB. ${ }^{5-7}$ Compared with the standard ALND, SLNB is associated with less arm and shoulder complications, such as pain, lymphedema, and sensory loss. ${ }^{8,9}$ 
The introduction of SLNB is based on the theory that SLN is the first lymph node for regional lymph drainage from a primary tumor site. According to that theory, tumor cells must pass through SLN before they arrive at the regional lymph nodes. ${ }^{10}$ The SLN status is a key indicator for the prediction of regional lymphatic involvement. ${ }^{11}$ Most importantly, SLNB enables the rapid intraoperative diagnosis and facilitates the doctors to decide whether an ALND should be performed. ${ }^{12}$ Therefore, the intraoperative detection of SLN metastasis has become a hot research topic in recent years. Currently, cytokeratin immunohistochemistry (CK-IHC) and hematoxylin and eosin (HE) staining of consecutive frozen sections have been widely applied in diagnosing lymph node metastasis. ${ }^{13}$ However, CK-IHC requires a long staining time ${ }^{14,15}$ and the rapid frozen pathologic sections are relatively thick and often poorly stained with a false-negative rate of up to $10 \%-30 \% .{ }^{16}$ As a result, a clinically accurate and effective method for the detection of lymph node metastasis has become a key step for the wider use of SLNB. Before the clinical introduction of a novel SLNB-based intraoperative diagnostic technology, its sensitivity, specificity, positive and negative predictive values, overall accuracy, and particularly, false-negative rate must be well defined.

In our present study, by developing a novel approach to intraoperative diagnosis of SLN metastasis, we identified and evaluated the SLN involvement at the molecular level. Meanwhile, the new method was combined and compared with conventional tests to highlight its accuracy and sensitivity, so as to provide new evidence for its clinical application potential and to facilitate intraoperative decision making.

\section{Materials and methods}

\section{Patients and clinical assessments}

Two hundred and six SLNs from 124 patients with primary breast cancer treated in the Tianjin Medical University Cancer Institute and Hospital were enrolled in this study. The inclusion criteria of patients included: a) invasive ductal carcinoma confirmed by core needle biopsy or excisional biopsy; b) no clinical evidence of axillary lymph node involvement or distant metastasis; c) no previous axillary surgery; and d) no previous history of chemotherapy or radiotherapy. After the SLNs were removed, breast mastectomy as well as ALND were performed. Pathologic examinations of the primary sites, SLNs, and axillary lymph nodes were performed at the department of pathology after the surgeries. Informed consent was obtained from all patients and the research protocols were approved by the Ethics Committee of Tianjin Medical University Cancer Institute and Hospital. The clinicopathological data of the patients are listed in Table 1.

\section{Experimental kits and antibodies}

Immunomagnetic beads used in the study were purchased from Nanjing Nanoeast Biotech Co., (Nanjing, People's Republic of China). The spherical magnetic nanoparticles were coated with streptavidin, and the diameters were about $50 \mathrm{~nm}$. CELLection Pan Mouse IgG kit, Dynal MPC-S magnetic separator, and DNase I were purchased from Invitrogen (Carlsbad, CA, USA). Rat anti-human MOC-31 antibody was purchased from Dako Denmark A/S (Glostrup, Denmark) and the fluorescent-labeled antibodies including MOC-31-fluorescein isothiocyanate (FITC), CD44v6-FITC, MUC1-PE, and HER2-PERCP as well as their control antibodies were purchased from Santa Cruz Biotechnology Inc., (Dallas, TX, USA). CK7 and CKAE1/AE3 monoclonal antibodies, secondary antibody kit, and DAB color development kit were purchased from ZSGB-Bio (Beijing, People's Republic of China). RPMI-1,640 and fatal bovine serum (FBS) were purchased from HyClone (Logan, UT, USA), and $0.25 \%$ trypsin and collagenase I were purchased from Gibco (Uxbridge, UK).

\section{Surgical techniques}

To ensure the consistency of SLNB, all the operations were performed by the same senior surgeon, who had rich clinical experience. Sentinel lymph node dissection (SLND) and

Table I Comparison of the three diagnostic methods in SLN surgery

\begin{tabular}{llll}
\hline Method & HE staining & CK-IHC & IMS \\
\hline Number of SLN metastasis patients & $34 / / 24$ & $35 / I 24$ & $38 / I 24$ \\
Time & $30-40$ minutes & $1-2$ days & $60-70$ minutes \\
Cost (¥) & 780 & 500 & 250 \\
Sensitivity & $77.20 \%$ & $79.50 \%$ & $86.4 \%$ \\
Specificity & $100.0 \%$ & $100.0 \%$ & $94.7 \%$ \\
Accuracy & $91.90 \%$ & $92.70 \%$ & $93.5 \%$ \\
False-positive rate & $0.00 \%$ & $0.00 \%$ & $2.50 \%$ \\
False-negative rate & $22.70 \%$ & $20.4 \%$ & $13.6 \%$ \\
The negative predictive value & $88.89 \%$ & $89.89 \%$ & $95.5 \%$ \\
The positive predictive value & $100.0 \%$ & $100.0 \%$ & $93.0 \%$ \\
\hline
\end{tabular}

Abbreviations: SLN, sentinel lymph node; HE, hematoxylin and eosin; CK-IHC, cytokeratin immunohistochemistry; IMS, immunomagnetic separation. 
ALND were performed according to standard protocols. After the patients were anesthetized, $4 \mathrm{~mL}$ methylene blue (at a concentration of $1 \%$, diluted with normal saline) was injected into the subcutaneous tissue around the primary tumor or at the surface of the tumor using a $5 \mathrm{~mL}$ empty syringe. If biopsy had already been performed before or during surgery, methylene blue was injected into the subcutaneous tissues around the residual cavity wall after biopsy and at the surface of the residual cavity. The method and dosage were the same for tumors at different sites. Skin and subcutaneous tissues were dissected 10-15 minutes after methylene blue injection. The skin flap was routinely removed. Fats and connective tissues were dissected along the outer edge of the pectoralis major muscle parallel to the muscle bundle. After a blue-dyed lymphatic vessel became visible, dissection was performed along this lymphatic vessel toward the armpit until a blue-dyed lymph node was found. This lymph node was removed and labeled as SLN. Subsequently, standard or modified radical mastectomy for breast cancer was performed. If a breast-conserving surgery had to be performed, an alternative incision was made along the axillary skin folds for SLNB.

\section{SLN processing}

As shown in Figure 1, all operations were performed on the ultra-clean bench, using the method described by Tsujimoto et al: ${ }^{17}$ put the SLN on a clean culture dish, and cut it into four equal blocks using a sterile scalpel blade. These blocks were labeled as a, b, c, and d clockwise. Each block was further trisected into sections a', b', and c'.

\section{HE staining and CK-IHC of SLNs}

Section b' was frozen, and then three layers $(\alpha, \beta$, and $\gamma)$ were sliced for HE staining. For CK-IHC, two slides were obtained from each layer for the immunohistochemical detection using anti-CK7 (1:100) and anti-CKAE1/AE3 (1:150) antibodies. Two indicators (the percentage of positive cells over total cell numbers and the staining intensity of positive cells) were used to semi-quantitatively interpret the staining results. The scoring for the staining intensity was as follows: zero, no specific staining; one, light yellow; two, brownish yellow; and three, brown. In addition, the scoring for the percentage of positive cells among all the observed cells $(n=1,000)$ was as follows: one, $<10 \%$; two, 10\%-50\%; three, $51 \%-75 \%$; and four, $>75 \%$. The overall score, based on the product of these indicators, was divided into four levels: 0-3, negative; four and five, +; six and seven, ++; eight and above, +++; among which + to +++ indicated positive expressions.

\section{Immunomagnetic separation (IMS) of SLN-specific MOC-3I monoclonal antibody}

The sections a' and c' for IMS were put on a screen beneath which there was a sterile culture dish. The sections were

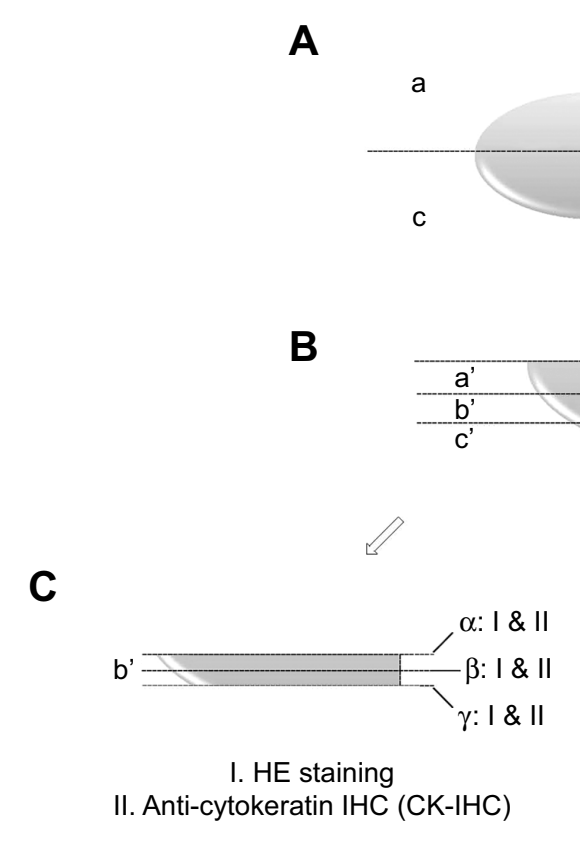

Figure I SLN prepared for different methods of intraoperative diagnosis.

Notes: (A) The SLN was cut into four equal blocks and label them as blocks a, b, c, and d clockwise; (B) Each block was further trisected into sections a', b', and c'; (C) The section b' of each block was frozen and stained with HE and CK-IHC, during which three layers ( $\alpha$, $\beta$, and $\gamma$ ) were sampled; (D) The sections a' and c' of each block underwent IMS.

Abbreviations: SLN, sentinel lymph node; HE, hematoxylin and eosin; CK-IHC, cytokeratin immunohistochemistry; IMS, immunomagnetic separation; IHC, immunohistochemistry. 
carefully cut into several small 1-2 mm pieces with ophthalmic scissors, and then ground. Single-cell suspension was prepared and repeatedly rinsed with $0.9 \%$ normal saline, and sterilized with a $0.22 \mu \mathrm{m}$ filter once. Next, the concentration of SLN single-cell suspension was adjusted to $10^{7}$ cells $/ \mathrm{mL}$. With a target cell-to-bead ratio of about $4: 1-8: 1$, the appropriate amount of beads were added for the pre-washing of immunomagnetic beads. The MOC-31 antibody was added into the single-cell suspension and labeled, and then added into the pre-washed beads. The beads thoroughly bound to the labeled rat anti-human MOC-31 antibody, became adherent in the magnetic field, and achieved cell separation on a magnetic particle concentrator (Dynal ${ }^{\circledR}$ MPC-S). Cell elution was then performed to collect MOC-31+-enriched single-cell suspension after IMS. Afterward, $1 \mathrm{~mL}$ of MOC-31+ single-cell suspension (cell count: 104) was collected for flow cytometry after having been labeled with rat anti-human MOC-31-FITC fluorescence antibody to evaluate the accuracy of the IMS of specific MOC-31 monoclonal antibody. The remaining MOC-31+ single-cell suspension was used for analyzing the expressions of CD44v6, MUC1, and HER2.

\section{Detection of CD44v6, MUCI, and HER2 in MOC-3I+-enriched cells}

The separated MOC-31+-enriched cells were labeled with CD44v6-FITC, MUC1-PE, and HER2-PERCP, respectively, before flow cytometry. Four control groups were set for the first analysis: a) labeled with the isotype control antibodies of these three antibodies; b) labeled with CD44v6-FITC and the isotype control antibodies of MUC1 and HER2; c) labeled with MUC1-PE and the isotype control antibodies of CD44v6 and HER2; and d) labeled with HER2-PERCP and the isotype control antibodies of CD44v6 and MUC1. The expressions of CD44v6, MUC1, and HER2 in MOC-31+ cells were defined as positive if CD $44 \mathrm{v} 6>50 \%, \mathrm{MUC} 1>50 \%$, and $\mathrm{HER} 2>20 \%$, among which the positive expression of one or more antibodies was interpreted as positive for lymph node metastasis.

\section{Follow-up}

The patients were followed up every 6 months after surgery. Clinical examination and imaging were performed at each follow-up visit. In the present study, a relapse of breast cancer refers to both the local recurrence and distant metastasis.

\section{Statistic analysis}

Data were analyzed using SPSS17.0 (SPSS Inc., Chicago, IL, USA). The comparison of the positive rate, negative rate, false and negative rate in detecting SLN metastasis among the SLN HE-stained group, CK-IHC group, and IMS group were performed using $\chi^{2}$-test. $\chi^{2}$-test was also applied for the comparison of inter-group rates and the analysis of clinicopathological features. $P<0.05$ was considered statistically significant difference.

\section{Results Detection of SLNs}

Among 124 patients with SLN, totally 206 SLNs were detected, including one SLN in 63 cases (50.8\%), two SLNs in 46 cases $(37.1 \%)$, three SLNs in nine cases $(7.3 \%)$, and four SLNs in six cases $(4.8 \%)$. SLNs were located at level I in 121 cases $(97.6 \%)$ and level II in three cases (2.4\%). After SLND, complete ALND was conducted in 124 patients who had undergone SLNB, during which a total of 2,163 axillary non-SLNs were detected. Axillary lymph node metastasis was noted in 44 patients, among whom 212 positive axillary lymph nodes were dissected.

\section{Histopathological study}

HE staining for 206 SLNs from 124 patients showed that 45 SLNs $(21.8 \%)$ from 34 patients had metastasis. Of 44 patients with positive axillary lymph nodes, HE staining of SLNs showed negative results in ten patients, yielding a false-negative rate of $22.7 \%$ (10/44). Therefore, HE staining of SLN had a sensitivity of $77.2 \%(34 / 44)$, specificity of $100 \%$ $(34 / 34)$, false-positive rate of $0 \%(0 / 34)$, accuracy of $91.9 \%$ (114/124), positive predictive value of $100 \%(34 / 34)$, and negative predictive value of $88.89 \%$ (80/90). CK-IHC of the 206 SLNs of 124 patients showed that 51 SLNs of 35 cases had metastasis. Of patients diagnosed to be with SLN metastasis by CK-IHC, CK7+ and CKAE1/AE3+ were found in 15 cases, $\mathrm{CK} 7+$ alone in 12 cases, and CKAE1/AE3+ alone in eight cases. Therefore, the CK-IHC for SLN metastasis had a sensitivity of $79.5 \%$ (35/44), specificity of $100.0 \%$ $(35 / 35)$, false-negative rate of $20.4 \%(9 / 44)$, accuracy of $92.7 \%(115 / 124)$, positive predictive value of $100 \%(35 / 35)$, and negative predictive value of $89.89 \%$ (80/89) (Table 1).

\section{IMS of SLN-specific MOC-3I monoclonal antibody}

After the SLNs harvested intraoperatively were prepared into single-cell suspensions, they were labeled with rat anti-human MOC-31 monoclonal antibody and underwent IMS. The obtained cells were labeled with rat anti-human MOC-31-FITC fluorescent antibody and then underwent flow cytometry, which yielded MOC-31+ cells with a purity of $93.62 \% \pm 2.86 \%(91.5 \%-98.7 \%)$ (Figure 2). Obviously, 
A

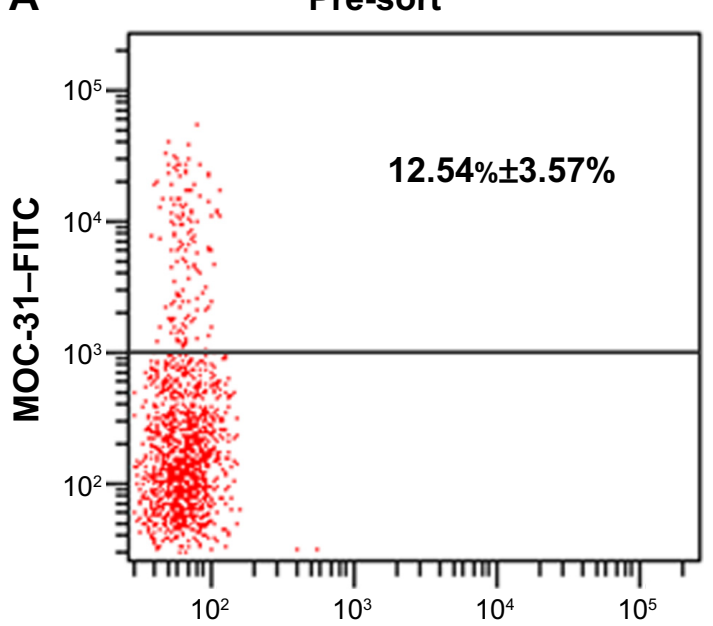

B

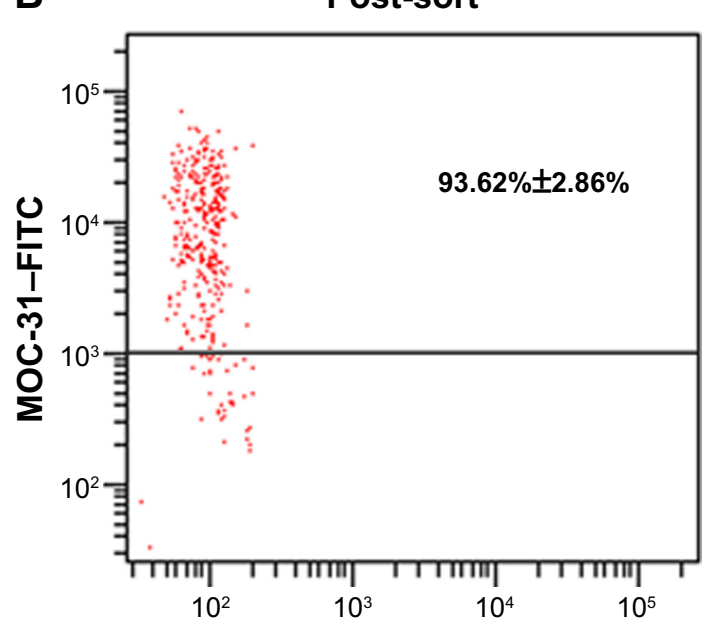

Figure 2 The different percentage of MOC-3I+ cells in SLN before and after immunomagnetic beads separation by flow cytometry.

Notes: After manufacturing single-cell suspension SLN, the cells were labeled with MOC-3I-FITC antibody. (A) The expression of MOC-3I in SLN cells before immunomagnetic beads separation and the percentage of MOC-3I+ cells is $12.54 \% \pm 5.57 \%$; (B) The expression of MOC-3I in SLN cells after immunomagnetic beads separation. After immunomagnetic beads separation, the percentage of MOC-3I+ cells is $93.62 \% \pm 2.86 \%$.

Abbreviations: SLN, sentinel lymph node; FITC, fluorescein isothiocyanate.

the IMS of specific MOC-31 monoclonal antibody can effectively enrich and separate the mo- $31+$ cells in the SLN single-cell suspension.

\section{Detection of SLN metastasis by determining the expressions of CD44v6, HER2, and MUCI with the combination of flow cytometry and IMS}

The single-cell suspension prepared by rat anti-human MOC-31 monoclonal antibody-labeled SLNs underwent IMS to separate the cells. Meanwhile, the expressions of three breast cancer-related markers MUC1, CD44v6, and HER2 were determined (Figure 3). One or more positive results among these three markers were interpreted as positive lymph node metastasis. Using the new method, 55 positive-SLN in 38 breast cancer patients were found, yielding a sensitivity of $86.4 \%$ (38/44), specificity of $94.7 \%$ (36/38), accuracy of $93.5 \%(116 / 124)$, false-positive rate of $2.5 \%(2 / 80)$, false-negative rate of $13.6 \%$ (6/44), positive predictive value of $95.5 \%$ (42/44), and negative predictive value of $93.0 \%(80 / 86)$.

\section{Comparison of the diagnostic values of three methods for the intraoperative detection of SLN metastasis}

As shown in Figure 4A and C, the IMS had higher diagnostic sensitivity and accuracy than HE staining and CK-IHC in detecting SLN metastasis $(P<0.001)$. Meanwhile, IMS had relatively lower false-negative rate and specificity than $\mathrm{HE}$ staining and CK-IHC $(P<0.001)$ (Figure 4B and D). Therefore, detection at single-cell level can remarkably reduce the false-negative results in the diagnosis of SLN metastasis and thus may avoid the painful second operation after SLNB. Besides, it required shorter intraoperative detection time and lower cost and had higher detection rate for micrometastasis (Table 1). Therefore, as an efficient and accurate method for the intraoperative detection of SLN metastasis in breast cancer patients, to some extent, IMS is feasible for clinical application.

\section{Expressions of CD44v6, HER2, and $\mathrm{MUCl}$ in SLNs of breast cancer patients and their correlation with the clinical features and prognosis}

As shown in our study, the expressions of these three tumor markers were positively correlated with the clinical features including the pathological stage of the primary lesion, cancer thrombus in the lymphatic vessel, and three-year relapse/ metastasis. Notably, the expressions of tumor markers in the SLNs of breast cancer patients were positively correlated with the number of metastatic axillary lymph nodes $(P=0.000)$ (Table 2). In patients with high expressions of CD44v6, MUC1, and HER2 in SLNs, the number of metastatic axillary lymph nodes was high in the postoperative frozen slides, among which the expressions of MUC1 and HER2 showed statistical significance $(P<0.05)$ (Table 3$)$. Therefore, the levels of these three tumor markers in SLNs are good indicators for predicting axillary lymph node 

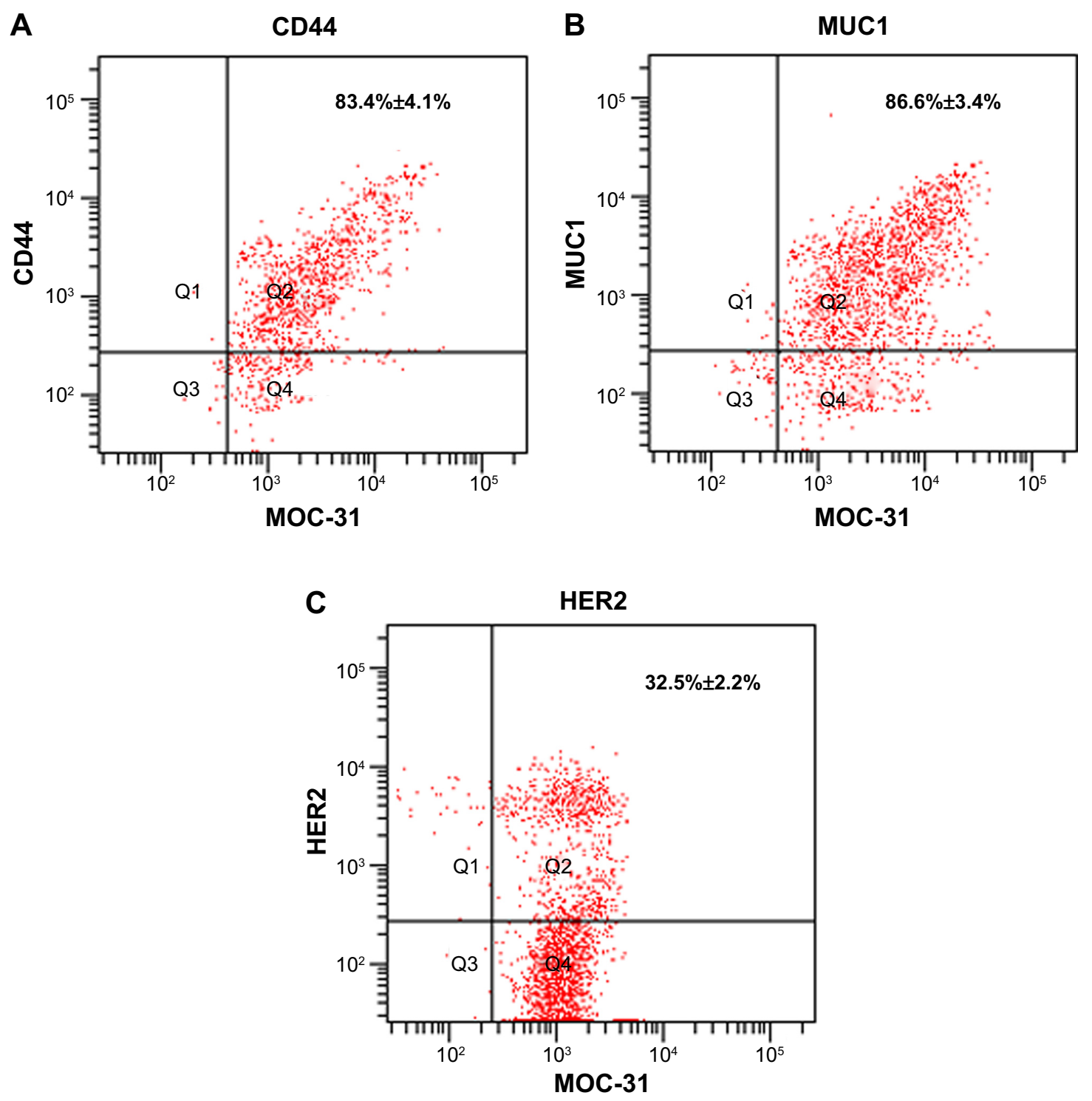

Figure 3 The expressions of CD44, MUCI, and HER2 in the magnetic bead separation sorted MOC-31+ cells by flow cytometry.

Notes: (A) The proportion of MOC-3I+CD44+ in the magnetic bead separation sorted MOC-3I+ cells were $83.4 \% \pm 4.1 \%$; (B) The proportion of MOC-3I+MUCI+ in the magnetic bead separation sorted MOC-3I+ cells were $86.6 \% \pm 3.4 \%$; (C) The proportion of MOC-3I+HER2+ in the magnetic bead separation sorted MOC-3I+ cells were $32.5 \% \pm 2.2 \%$.

metastasis in patients with breast cancer. For patients with large primary lesions and/or high tumor stages, the possibility of SLN metastasis must be cautiously considered, to facilitate tailored treatment.

\section{Discussion}

In breast cancer surgery, the shift from "maximum tolerable" to "minimum effective" treatment has been evident at the beginning of this century. This trend represents the change in treatment methods, and more importantly, it embodies a major development of the principles and concepts in the surgical treatment of breast cancer toward a comprehensive system based on the existing standard protocols, which will incorporate individualized, minimally invasive, accurate approaches, as well as protection and focus on physical and psychological rehabilitation.

The rapid development of SLNB techniques is a concrete manifestation of such concept under clinical settings. As a minimally invasive diagnostic technique, SLNB identifies the presence of lymph node metastasis without extensive injury to patients, serving as guidance for conducting complete ALND. At present, HE staining for frozen sections in combination with CK-IHC are often used for intraoperative SLN detection. ${ }^{12}$ However, the former method is associated with a high false-negative rate due to thick sections and poor staining, while a wide application of CK-IHC staining is 
A

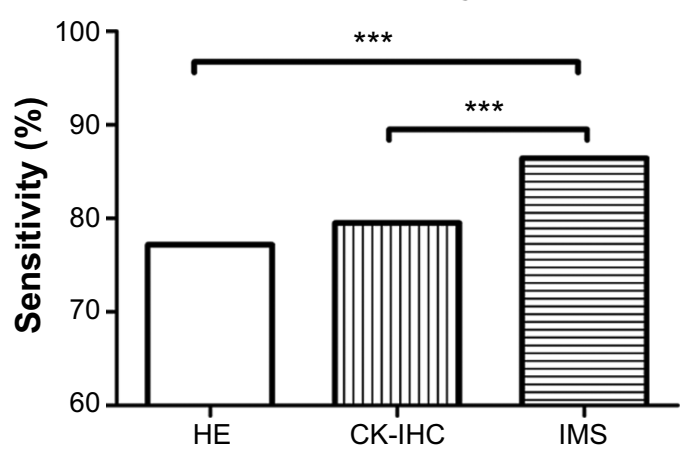

C

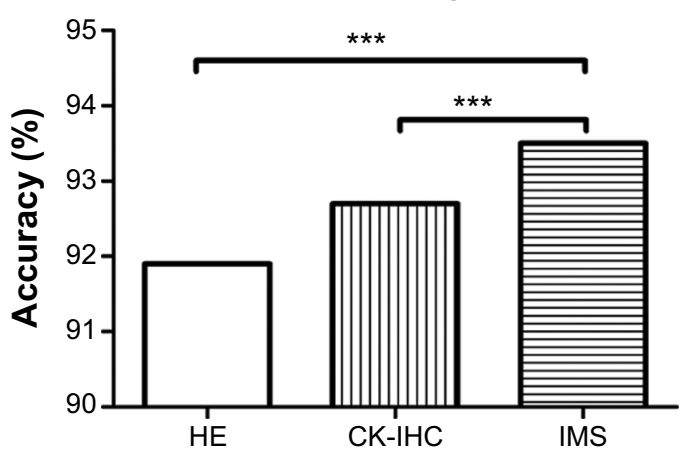

B

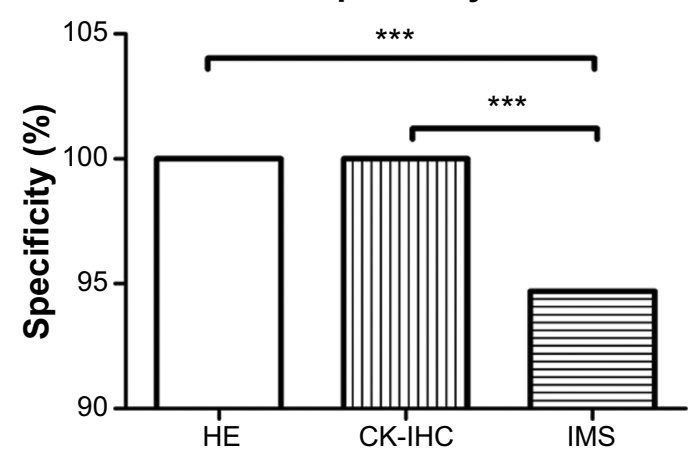

D

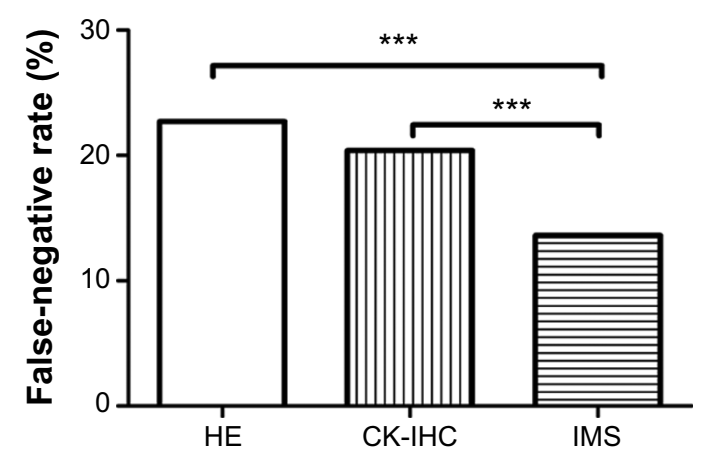

Figure 4 The diagnosis value of SLN metastasis by different methods (HE, CK-IHC, and IMS).

Notes: (A) The sensitivity in diagnosis of SLN metastasis; (B) The specificity in diagnosis of SLN metastasis; (C) The accuracy in diagnosis of SLN metastasis; (D) The falsenegative rate in diagnosis of SLN metastasis. The statistical analysis was performed with Student's $t$-test. $* * *: P<0.00$ I compared with the IMS group.

Abbreviations: SLN, sentinel lymph node; HE, hematoxylin and eosin; CK-IHC, cytokeratin immunohistochemistry; IMS, immunomagnetic separation.

difficult because of its time-consuming nature. ${ }^{16}$ Therefore, it is necessary to develop a more efficient and feasible intraoperative detection technology for clinical application.

The IMS technology is a new immunological approach of interest among global investigators in recent years. Immunomagnetic beads are magnetic microspheres coated with monoclonal antibodies, which are used to form new complexes by binding to target substances containing the corresponding antigen. When passing through a magnetic field, these complexes are detained and separated with the other components, a procedure known as IMS. Due to its high specificity, high concentration and high separation rate without interference with cell activities, the IMS method is useful in identifying an extremely small amount of tumor cells from massive peripheral blood cells. When used in combination, this method makes up for the deficiencies of conventional detection techniques (such as immunocytochemistry, RT-PCR, and flow cytometry), and improves the detection sensitivity and specificity for circulating tumor cells. Up to now, the IMS method has been used to detect peripheral blood tumor cells from patients with many different cancers and other conditions, ${ }^{18-20}$ and its value in the detection of circulating tumor cells has been recognized. However, it has not been employed in SLN biopsies. An epithelial/ adenocarcinoma marker, MOC-31 has been commonly reported in recent years, of which the expression was originally found in the GLS-1 small cell lung cancer cell lines. Subsequent histological studies confirm its expression in almost all kinds of adenocarcinoma, ${ }^{21,22}$ and it serves as a critical immunological marker of metastatic adenocarcinoma. ${ }^{23}$ In the present study, the SLN single-cell suspension was marked with mouse anti-human MOC-31 antibodies for IMS. MOC-31 antibodies were capable of labeling the metastatic breast cancer cells in SLNs before IMS, so that the enriched cells will maintain purity and activity.

MUC1 is an important member of the mucin family, which is associated with a variety of tumors. The expression of MUC1 has been found in normal breast and tumor tissues, ${ }^{24,25}$ though the reported expression levels varied in different breast lesions. Due to incomplete and premature glycosylation of MUC1 in tumor tissues, new protein epitopes or carbohydrate antigens of the core protein are 
Table 2 Baseline characteristics of 124 patients with early stage breast cancer according to CD44v6, HER2, and MUCI status of SLNs

\begin{tabular}{|c|c|c|c|c|c|c|c|}
\hline \multirow[t]{3}{*}{ Characteristics } & \multirow{3}{*}{$\begin{array}{l}\text { Total } \\
\text { number }\end{array}$} & \multicolumn{4}{|c|}{ Status of SLNs } & \multirow[t]{3}{*}{$x^{2}$} & \multirow[t]{3}{*}{$P$} \\
\hline & & \multicolumn{2}{|c|}{$\begin{array}{l}\text { CD44v6+/HER2+/MUCI+ } \\
\mathrm{N}=38\end{array}$} & \multicolumn{2}{|c|}{$\begin{array}{l}\text { CD44v6-/HER2-/MUCI- } \\
\mathrm{N}=86\end{array}$} & & \\
\hline & & Number & $\%$ & Number & $\%$ & & \\
\hline Age, years & & & & & & 0.027 & 0.869 \\
\hline$\leq 35$ & 25 & 8 & 21.1 & 17 & 19.7 & & \\
\hline$\geq 35$ & 99 & 30 & 78.9 & 69 & 80.3 & & \\
\hline Axillary lymph node status & & & & & & 84.029 & 0.000 \\
\hline Negative & 80 & 2 & 5.3 & 78 & 90.7 & & \\
\hline Positive & 44 & 36 & 94.7 & 8 & 9.3 & & \\
\hline Size $(\mathrm{cm})$ & & & & & & 0.356 & 0.546 \\
\hline$\leq 2 \mathrm{~cm}$ & 45 & 12 & 31.6 & 32 & 37.2 & & \\
\hline$>2 \mathrm{~cm}$ & 79 & 26 & 68.4 & 54 & 63.8 & & \\
\hline Grade & & & & & & 17.060 & 0.000 \\
\hline I & 31 & 1 & 2.6 & 30 & 34.9 & & \\
\hline$\|$ & 89 & 34 & 89.5 & 55 & 64.0 & & \\
\hline III & 4 & 3 & 7.9 & 1 & 1.1 & & \\
\hline Lymphovascular invasion & & & & & & 15.434 & 0.000 \\
\hline No & 103 & 24 & 63.2 & 79 & 91.9 & & \\
\hline Yes & 21 & 14 & 36.8 & 7 & 8.1 & & \\
\hline Hormones & & & & & & 0.969 & 0.325 \\
\hline Negative & 35 & 13 & 34.2 & 22 & 25.6 & & \\
\hline Positive & 89 & 25 & 65.8 & 64 & 74.4 & & \\
\hline HER2 & & & & & & 80.262 & 0.609 \\
\hline Negative & 29 & 28 & 73.7 & 67 & 22.1 & & \\
\hline Positive & 95 & 10 & 26.3 & 19 & 77.9 & & \\
\hline 3 years recurrence/metastasis & & & & & & 14.872 & 0.000 \\
\hline No & 113 & 29 & 76.3 & 84 & 97.6 & & \\
\hline Yes & 11 & 9 & 23.7 & 2 & 2.3 & & \\
\hline
\end{tabular}

Abbreviation: SLN, sentinel lymph node.

exposed and distributed on the entire surface of cancer cells, which can be recognized as the tumor-specific antigens by the immune system. ${ }^{26}$ Overexpression of MUC1 is observed in over $90 \%$ breast cancer. ${ }^{27}$ Hamada et al demonstrated in their study that the MUC1 expression was related to lymph node metastasis. ${ }^{28}$ The human CD44 gene is located on the short arm of chromosome 11 , and consists of 20 exons. CD44 can be classified as CD44s or CD44v depending on the composition of the exons encoding CD44. As a cell surface adhesion molecule, CD44v6 is involved in the specific adhesion between cells and the extracellular matrix. It may mediate the binding between the lymphocytes and vascular endothelial

Table 3 The expression of CD44v6, MUCI, and HER2 in the patient of positive axillary lymph node

\begin{tabular}{|c|c|c|c|c|c|c|c|}
\hline \multirow[t]{3}{*}{ Status of SLN } & \multirow[t]{3}{*}{ Total number } & \multicolumn{4}{|c|}{ Axillary lymph node status $N=44$} & \multirow[t]{3}{*}{$\chi^{2}$} & \multirow[t]{3}{*}{$P$} \\
\hline & & $\mathbf{N}=\mathbf{3 8}$ & & \multicolumn{2}{|c|}{$\begin{array}{l}>3 \text { nodes positive } \\
\mathrm{N}=6\end{array}$} & & \\
\hline & & Number & $\%$ & Number & $\%$ & & \\
\hline CD44v6 & & & & & & 3.889 & 0.049 \\
\hline $50 \%-80 \%$ & 30 & 28 & 71.05 & 2 & 33.33 & & \\
\hline$>80 \%$ & 14 & 10 & 28.95 & 4 & 66.67 & & \\
\hline $\mathrm{MUCl}$ & & & & & & 11.008 & 0.001 \\
\hline $50 \%-80 \%$ & 32 & 31 & 96.86 & 1 & 16.67 & & \\
\hline$>80 \%$ & 12 & 7 & 3.14 & 5 & 83.33 & & \\
\hline HER2 & & & & & & 4.599 & 0.032 \\
\hline $20 \%-30 \%$ & 31 & 29 & 76.32 & 2 & 33.33 & & \\
\hline$>30 \%$ & 13 & 9 & 23.68 & 4 & 66.67 & & \\
\hline
\end{tabular}

Abbreviation: SLN, sentinel lymph node. 
cells, facilitating the return of lymphocytes to lymphoid tissues through the vascular walls. Therefore, it is also referred as the homing receptor. Many studies have shown that CD44v6 is related with lymph node metastasis, and is one of the important indicators for micrometastasis..$^{29,30}$ Oncogene HER2 is located on the long arm of chromosome 17, whose protein products are highly homologous to the epidermal growth factor receptor. HER2 expression is related to the differentiation and proliferation of tumor cells, and the response and prognosis of endocrine treatment, though the relationship with the histological type of breast cancer, tumor size, and lymph node metastasis remains controversial. Therefore, MUC1, CD44v6, and HER2 are all the targets related to lymph node metastasis of breast cancer.

In the present study, we performed SLNB using the methylene blue method, and the resultant SLNs were prepared into single-cell suspensions. Flow cytometry analysis was conducted to determine the expression levels of the three metastasis-related markers in MOC-31 positive cells collected by IMS. Compared with the intraoperative frozen SLN sections commonly used in clinical practice, IMS and flow cytometry analysis for metastasis-related factors have higher sensitivity and accuracy for intraoperative SLN diagnosis, which indicates the potential to evaluate the status of axillary lymph node metastasis more accurately. However, the IMS technology also exhibits slightly lower specificity and a positive predictive value. The deficiency may be related to the affinity of the MOC-31 antibody coupled to the immunomagnetic beads, the specificity of the antibodies used, and the significance of the breast cancer metastasis-related biomarkers (CD44v6, MUC1, and HER2). However, with the development of modern science and technology, better and more specific immunomagnetic beads and antibodies will be prepared. Meanwhile, more representative and significant breast cancer metastasis-related biomarkers will be determined; subsequently, the specificity and the positive predictive value of IMS technology may well be improved and therefore become superior. Therefore, the diagnostic model established in this study is expected to provide new ideas for reforming existing intraoperative SLN diagnostic methods.

\section{Conclusion}

In conclusion, the IMS technique enables accurate detection with high detection rate, specificity and sensitivity, low falsenegative rate, short detection time, less cost, and relatively simple operating conditions. In view of the correlation with the clinical and pathological characteristics of patients in our preliminary findings, further studies can be performed to identify new treatment and prognosis prediction for breast cancer by evaluating the prognostic value. Although we used a breast cancer model, IMS technology may also be applied for the detection of lymph node metastasis in other cancers if only the corresponding lymph node metastasis-related markers are identified. Therefore, IMS technology might be a potential new method in cancer lymph node metastasis detection in the future.

\section{Acknowledgments}

This study was supported by the National Science and Technology Support Program (No 2013BAI09B08) and Tianjin Municipal Major Scientific and Technological Special Project for Significant Anticancer Development (No 12ZCDZSY15700).

\section{Author contributions}

All authors contributed substantially to the concept and design of the study, drafting of the article, and critical revision of the manuscript. All authors have read and approved the final version of the manuscript for publication.

\section{Disclosure}

The authors report no conflicts of interest in this work.

\section{References}

1. Koller M, Barsuk D, Zippel D, Engelberg S, Ben-Ari G, Papa MZ. Sentinel lymph node involvement - a predictor for axillary node status with breast cancer - has the time come? Eur J Surg Oncol. 1998;24(3):166-168.

2. Ahmed M, Purushotham AD, Douek M. Novel techniques for sentinel lymph node biopsy in breast cancer: a systematic review. Lancet Oncol. 2014;15(8):e351-e362.

3. Singletary SE. Systemic treatment after sentinel lymph node biopsy in breast cancer: who, what, and why? J Am Coll Surg. 2001;192(2): 220-230.

4. Land SR, Kopec JA, Julian TB, et al. Patient-reported outcomes in sentinel node-negative adjuvant breast cancer patients receiving sentinelnode biopsy or axillary dissection: National Surgical Adjuvant Breast and Bowel Project phase III protocol B-32. J Clin Oncol. 2010;28(25): 3929-3936

5. Giuliano AE, McCall L, Beitsch P, et al. Locoregional recurrence after sentinel lymph node dissection with or without axillary dissection in patients with sentinel lymph node metastases: the American College of Surgeons Oncology Group Z0011 randomized trial. Ann Surg. 2010; 252(3):426-432.

6. Lyman GH, Giuliano AE, Somerfield MR, et al. American Society of Clinical Oncology guideline recommendations for sentinel lymph node biopsy in early-stage breast cancer. J Clin Oncol. 2005;23(30):7703-7720.

7. Veronesi U, Paganelli G, Viale G, et al. A randomized comparison of sentinel-node biopsy with routine axillary dissection in breast cancer. N Engl J Med. 2003;349(6):546-553.

8. Krag D, Weaver D, Ashikaga T, et al. The sentinel node in breast cancera multicenter validation study. N Engl J Med. 1998;339(14):941-946.

9. Kuwajerwala NK, Feczko C, Dekhne N, et al. Comparison of lymphedema in patients with axillary lymph node dissections to those with sentinel lymph node biopsy followed by immediate and delayed ALND. Am J Clin Oncol. 2013;36(1):20-23. 
10. Mansel RE, Fallowfield L, Kissin M, et al. Randomized multicenter trial of sentinel node biopsy versus standard axillary treatment in operable breast cancer: the ALMANAC Trial. J Natl Cancer Inst. 2006;98(12): 599-609.

11. Schwartz GF, Giuliano AE, Veronesi U, et al. Proceedings of the consensus conference on the role of sentinel lymph node biopsy in carcinoma of the breast. Breast J. 2002;8:126-138.

12. Harlow SP, Krag DN, Julian TB, et al. Prerandamization Surgical Training for National Surgical Adjuvant Breast and Bowel Project (NSABP) B-32 trial, a randomized phase III clinical trial to compare sentinel node resection to conventional axillary dissection in clinically node-negative breast cancer. Ann Surg. 2005;241(1):48-54.

13. Treseler P. Pathologic examination of the sentinel lymph node: what is the best method? Breast J. 2006;12(suppl 2):S143-S151.

14. Pargaonkar AS, Beissner RS, Snyder S, et al. Evaluation of immunohistochemistry and multiple-level sectioning in sentinel lymph nodes from patients with breast cancer. Arch Pathol Lab Med. 2003;127(6): 701-705.

15. Weaver DL, Krag DN, Manna EA, et al. Detection of occult sentinel lymph node micrometastases by immunohistochemistry in breast cancer: an NSABP Protocol B-32 Quality Assurance Study. Cancer. 2006; 107(4):661-667.

16. Wada N, Imoto S, Hasebe T, et al. Evaluation of intraoperative section diagnosis of sentinel lymph nodes in breast cancer. Jpn J Clin Oncol. 2004;34(3):113-117.

17. Tsujimoto M, Nakabayashi K, Yoshidome K, et al. One-step nucleic acid amplification for intraoperative detection of lymph node metastasis in breast cancer patients. Clin Cancer Res. 2007;13(16):4807-4816.

18. Kim SJ, Ikeda N, Shiba E, Takamura Y, Noguchi S. Detection of breast cancer micrometastases in peripheral blood using immunomagnetic separation and immunocytochemistry. Breast Cancer. 2001;8(1):63-69.

19. Nakamura T, Yasumura T, Hayashi K, et al. Immunocytochemical detection of circulating esophageal carcinoma cells by immunomagnetic separation. Anticancer Res. 2000;20(6C):4739-4744.

20. Oyaizu N, Kozai Y, Kodo H, et al. A case of pure red cell aplasia complicated with diffuse large B cell lymphoma, T-cell-rich/histiocyte-rich variant: effectiveness of rituximab and implications for a common immunopathogenic role of B lymphocytes. Acta Haematol. 2005; 113(3):194-197.
21. Pai RK, West RB. MOC-31 exhibits superior reactivity compared with Ber-EP4 in invasive lobular and ductal carcinoma of the breast: a tissue microarray study. Appl Immunohistochem Mol Morphol. 2009;17(3): 202-206.

22. Pu RT, Pang Y, Michael CW. Utility of WT-1, p63. MOC31, mesothelin, and cytokeratin (K903 and CK5/6) immunostains in differentiating adenocarcinoma, squamous cell carcinoma, and malignant mesothelioma in infusion. Diagn Cytopathol. 2007;36(1):20-25.

23. Kim MJ, Shin HC, Shin KC. Best immunohistochemical panel in distinguishing adenocarcinoma from squamous cell carcinoma of lung: tissue microarray assay in resected lung cancer specimens. Ann Diagn Pathol. 2013;17(1):85-90.

24. Pargaonkar AS, Beissner RS, Snyder S, Speights VO Jr. Evaluation of immunohistochemistry and multiple-level sectioning in sentinel lymph nodes from patients with breast cancer. Arch Pathol Lab Med. 2003; 127(6):701-705.

25. Apostolopoulos V, Pietersz GA, McKenzie IF. MUC1 and breast cancer. Curr Opin Mol Ther. 1999;1(1):98-103.

26. Senapati S, Das S, Batra SK. Mucin-interacting proteins: from function to therapeutics. Trends Biochem Sci. 2010;35(4):236-245.

27. Kufe DW. Mucins in cancer: function, prognosis and therapy. Nat Rev Cancer. 2009;9(12):874-885.

28. Hamada T, Nomura M, Kamikawa Y, et al. DF3 epitope expression on MUC1 mucin is associated with tumor aggressiveness, subsequent lymph node metastasis, and poor prognosis in patients with oral squamous cell carcinoma. Cancer. 2012;118(21):5251-5264.

29. Okayama H, Kumamoto K, Saitou K, et al. CD44v6, MMP-7 and nuclear $\mathrm{Cdx} 2$ are significant biomarkers for prediction of lymph node metastasis in primary gastric cancer. Oncol Rep. 2009;22(4):745-755.

30. Tempfer C, Lösch A, Heinzl H, et al. Prognostic value of immunohistochemically detected CD44 isoforms CD44v5, CD44v6 and CD44v7-8 in human breast cancer. Eur J Cancer. 1996;32A(11):2023-2025.
International Journal of Nanomedicine

\section{Publish your work in this journal}

The International Journal of Nanomedicine is an international, peerreviewed journal focusing on the application of nanotechnology in diagnostics, therapeutics, and drug delivery systems throughout the biomedical field. This journal is indexed on PubMed Central, MedLine, CAS, SciSearch ${ }^{\circledR}$, Current Contents ${ }^{\circledR} /$ Clinical Medicine,

\section{Dovepress}

Journal Citation Reports/Science Edition, EMBase, Scopus and the Elsevier Bibliographic databases. The manuscript management system is completely online and includes a very quick and fair peer-review system, which is all easy to use. Visit http://www.dovepress.com/ testimonials.php to read real quotes from published authors. 